\title{
Identification of vowels in "vowelless" syllables by 3-year-olds
}

\author{
WALTER D. MURPHY, SANDRA L. SHEA, and RICHARD N. ASLIN \\ University of Rochester, Rochester, New York
}

\begin{abstract}
The ability of 3-year-old children to perceive the identity of vowels in full-vowel and silentcenter, consonant-vowel-consonant (CVC) syllables was investigated using a two-alternative pointing procedure. Silence replaced the middle $10 \%, 35 \%, 65 \%$, or $90 \%$ of the steady-state formants of synthetic "bad" and "bud" syllables. Identification of the two full-vowel syllables was 87\% correct, whereas performance for the silent-center syllables was somewhat lower $(72 \%, 70 \%, 67 \%$, and $66 \%$ correct for the $10 \%, 35 \%, 65 \%$, and $90 \%$ deletion stimuli, respectively). The performance of individual children fell into two subgroups: (1) those who performed like adults by maintaining correct vowel identification for all of the silent-center syllables, and (2) those who identified the full-vowel syllables correctly but performed at chance for all of the silent-center syllables. Three additional experiments showed that none of the children performed poorly when noise replaced the gap in the silent-center syllables.jThese results demonstrate that many 3-year-olds can identify vowels correctly in CVC syllables in the absence of the full spectral properties of steady-state formants.
\end{abstract}

The information contained in the quasi-steady-state formants of natural or synthetic speech tokens is sufficient for reliable vowel identification by adults (Delattre, Liberman, Cooper, \& Gerstman, 1952; Joos, 1948). However, this quasi-steady-state information varies across talkers (Peterson \& Barney, 1952) and across consonant contexts (Strange, Verbrugge, Shankweiler, \& Edman, 1976; Verbrugge, Strange, Shankweiler, \& Edman, 1976). Despite these context dependencies, adult listeners can identify vowels correctly when they are produced in isolation by different talkers (Diehl, McCusker, \& Chapman, 1981). However, when vowels are cross-spliced from one context to another, vowel identification becomes very poor (Kuwabara, 1985). These results provide support for theories that propose a role for dynamic formant information in vowel perception.

Additional support for the importance of dynamic formant information in vowel perception comes from studies of "vowelless" syllables. Adults can reliably identify vowels in consonant-vowel-consonant (CVC) syllables even when up to $90 \%$ of the middle of the syllable has been replaced with either silence or noise (Jenkins, Strange, \& Edman, 1983; Parker \& Diehl, 1984; Strange, Jenkins, \& Johnson, 1983), thereby eliminating any quasi-

\footnotetext{
This research was supported by NIH Research Grant HD-20286. During the preparation of this article, $\mathbf{R}$. N. Aslin was supported in part by the Center for Advanced Study in the Behavioral Sciences, by NSF Grant BNS 87-00864 to the Center, and by a fellowship from the John Simon Guggenheim Memorial Foundation. The assistance of Jill Gallipeau, Suzanne Holland, Brenda McGriff, and Carla Monestere is gratefully acknowledged. David Pisoni, Jerry Forshee, and David Link provided invaluable technical assistance. Reprint requests should be addressed to Richard N. Aslin, Department of Psychology, University of Rochester, Rochester, NY 14627.
}

steady-state formant information. Moreover, the vowelduration differences that are present in naturally produced CVC syllables (i.e., the short vowels $/ \mathrm{l} /, / \varepsilon /, \mid \mathrm{N} /, / \mathrm{u} / \mathrm{vs}$. the long vowels $/ e /, / æ /, / a /, / o /)$ are not necessary for correct vowel identification in vowelless syllables (Strange et al., 1983). Thus, two types of information contained in the formant transitions at syllable onset and syllable offset could account for adults' ability to identify correctly the vowels in vowelless CVC syllables: (1) the configuration of the formant transitions themselves (i.e., their starting frequencies and rate of change), and/or (2) the mid-syllable formant-frequency "targets" interpolated from the trajectories of the formant transitions.

These findings from adults raise several issues concerning the development of vowel perception. First, because vowel perception in adults is neither categorical nor based upon universal regions within the F1-F2 vowel space, it seems likely that the partitioning of the vowel space requires some minimal listening experience in a nativelanguage environment. Similarly, the ability to discount individual acoustic variations across talkers and across consonantal contexts may require considerable postnatal exposure. Second, although several studies of infants have indicated that natural vowel tokens can be categorized correctly despite variations in pitch contour and talker (Kuhl, 1979,1983 ), the stimuli in these studies were not CVC syllables and they contained only quasi-steady-state formant information. Thus, the ability of infants to categorize vowels on the basis of dynamic formant information has not been investigated. Because coarticulation effects in fluent speech reduce both the duration of quasi-steadystate formant information and the formant-frequency targets for vowels, infants and young children may rely more heavily than adults on invariant quasi-steady-state formant 
information than on variable dynamic formant information.

In the present series of experiments, a two-alternative forced-choice procedure was used to examine 3-year-olds' identification of vowels embedded in synthetic CVC syllables. The methodology used to obtain these identification data was very similar to that used by Kubaska and Aslin (1985) in a study of 3-year-olds' categorization of isolated vowels produced by several talkers. In that study, children were taught a two-alternative pointing task in which a correct response triggered a visual reward. The two training stimuli were natural tokens of the vowels /æ/ and $/ \mathrm{N}$ produced by a male adult. When consistent pointing responses were shown to these training stimuli, trials on which one of the same two vowels produced by a female adult, a male child, or a female child were presented. Children received differential reinforcement to generalize their pointing from the adult male tokens to the tokens produced by the other talkers. Not only did they quickly and consistently learn to generalize their responses, but they failed to learn an arbitrary grouping (that is, one not based on vowel quality). Because some of the vowel tokens overlapped in the F1-F2 vowel space, these results suggest that vowel normalization is operative by 3 years of age.

The methodology used by Kubaska and Aslin (1985) was modified slightly in the present series of experiments to obtain identification rather than categorization data from 3-year-olds. Children were trained on the two-alternative pointing task with the two full-vowel CVC syllables. Following training, several test stimuli in which a portion of the vowel was replaced with silence or noise were presented on separate trials. For these test stimuli, the procedure differed from that of Kubaska and Aslin (1985) in that no differential reinforcement was delivered. However, differential reinforcement was maintained for the original training stimuli, which were randomly interleaved into the test-trial sequence. Because young children will not remain responsive if reinforcement is discontinued, differential reinforcement was eliminated for the test stimuli by reinforcing the initial pointing response regardless of direction. This procedure prevented the children from being trained to associate certain test stimuli with one of the two full-vowel syllables, and therefore is analogous to a two-alternative forced-choice identification task without feedback commonly used to test adults.

\section{EXPERIMENT 1}

It was not clear how well children would generalize their responding from full-vowel CVC syllables to silent-center
CVC syllables, particularly if all of the quasi-steady-state formant information were eliminated. Therefore, a graded series of silent-center syllables was constructed. As in past studies of adults, the silent-center syllables were created by removing a portion of the middle of a full-vowel syllable and replacing that portion with silence. In contrast to previous studies, the full-vowel syllables were generated synthetically rather than using natural-speech syllables. An advantage associated with using synthetic speech is the ability to control the characteristics of the formant transitions, including their onset and offset frequencies and their durations. Although analyses of the edited natural-speech syllables used in previous studies verified that insertion of silence prevented the formant transitions from reaching their asymptotic frequencies and peak amplitude, neither formant onset and offset frequencies nor formant durations were held constant across syllables. Thus, the rate of change in formant frequencies and the configuration (frequency, differences) of formant transitions were uncontrolled. A disadvantage associated with using synthetic speech is that by eliminating the contextconditioned variability contained in natural speech, some critical combination of "cues" to vowel identity may be reduced or eliminated. On the one hand, therefore, use of synthetic speech may make the children's task of identifying vowels in silent-center syllables more difficult because some context-conditioned cues are eliminated (e.g., differences in formant-frequency onset and offset). On the other hand, the use of synthetic speech may make the task easier because for all but the longest duration silent-center syllables, some steady-state formant information is available for vowel identification.

\section{Method}

Subjects. The child subjects were 3-year-olds recruited as siblings of infants tested in other studies or through a newspaper advertisement. Their parents were contacted by letter and a follow-up telephone call. The subjects were tested over two to five sessions and were paid $\$ 3$ per visit. Of 16 subjects tested, 11 succeeded in reaching the final stage of the procedure and provided usable data. Of those who were unsuccessful, 4 were unable to learn the task and 1 left the study prematurely due to parental scheduling conflicts. Successful subjects ranged in age from 36.3 to 44.5 months with a mean age of $\mathbf{4 0 . 9}$ months. Three adult subjects provided baseline data for comparison to the children.

Stimuli. The stimuli were the CVC syllables /bæd/ and /bnd/ generated on the Klatt (1980) software synthesizer. Table 1 lists the parameters used to generate these stimuli, which were each $300 \mathrm{msec}$ in duration and had a characteristic adult-male voice quality. Fundamental frequency was $120 \mathrm{~Hz}$ for the initial $120 \mathrm{msec}$ and fell to $100 \mathrm{~Hz}$ over the final $180 \mathrm{msec}$. The initial and final formant transitions for both stimuli were $\mathbf{4 0}$ and $60 \mathrm{msec}$, respectively. A waveform editing program (Luce \& Carrell, 1981) was used to construct four additional versions of each syllable in which

Table 1

Formant-Frequency Values for Stimuli in Experiment 1

\begin{tabular}{|c|c|c|c|c|c|c|c|c|c|}
\hline \multirow[b]{2}{*}{ Stimulus } & \multicolumn{3}{|c|}{ F1 } & \multicolumn{3}{|c|}{$\mathbf{F} 2$} & \multicolumn{3}{|c|}{ F3 } \\
\hline & Start & Steady-State & End & Start & Steady-State & End & Start & Steady-State & End \\
\hline /bæd/ & 270 & 620 & 270 & 1186 & 1660 & 1850 & 2149 & 2430 & 2560 \\
\hline /bnd/ & 270 & 620 & 270 & 1186 & 1220 & 1850 & 2149 & 2550 & 2560 \\
\hline
\end{tabular}


the central segment of the vowel was replaced with silence. The 200-msec segment of each syllable between the initial and final formant transitions contained 22 pitch periods. Replacement of 2, 8, 14 , or 20 of these pitch periods with silence resulted in tokens with approximately $10 \%, 35 \%, 65 \%$, and $90 \%$ of the steady-state vowel deleted (see Figure 1). Pitch periods were deleted at a zero crossing in the waveform to eliminate transients. Stimulus intensities for the full-vowel through $90 \%$ deletion syllables ranged from 60 to $53 \mathrm{~dB}$ SPL (C-scale) measured with a General Radio sound-level meter at the approximate location of the child's head.

Apparatus. Testing was conducted in an IAC single-wall soundattenuated booth $(8 \times 8 \mathrm{ft})$. The child was seated (alone or in a parent's lap) in a chair facing a loudspeaker (Radio Shack MC-1000) located slightly above the level of the child's head. Two 13-in. color television sets (RCA XL-100) were located approximately $45^{\circ}$ to the child's left and right. A videodisc player (RCA Selectavision) was gated under computer control to the two TVs. A camera inside the booth allowed an experimenter outside the booth to monitor the subject's pointing responses. This experimenter entered subject data and operated the computer program that controlled the experiment. Stimulus presentation and reinforcement were controlled by a $\mathrm{PDP}_{\bar{F}}$ $11 / 34$ computer via a keyboard and button box. The stimuli were presented on-line through a 12-bit D/A converter with a sampling rate of $10 \mathrm{kHz}$ after low-pass filtering at $4.8 \mathrm{kHz}$. The apparatus was similar to that shown schematically in Figure 2 of Kubaska and Aslin (1985).

Procedure. After selecting a movie to serve as the visual reward, the child and parent entered the testing booth and sat in a chair positioned across from the loudspeaker. A second experimenter entered the booth and sat in front of the parent and child to assist the child in choosing the correct television for each training stimulus. This second experimenter was positioned beneath the loudspeaker and halfway between the two TVs so that neither the sounds nor the visual rewards were occluded during training. The entire experimental procedure consisted of three phases: shaping, testing, and identification.

In the shaping phase, Experimenter 1 (outside the booth) initiated the presentation of the full-vowel training syllables and coded the side to which the child pointed by pressing buttons sensed by the computer. All syllables were presented with an interstimulus interval of 500 msec. A response was judged to be "correct' by the computer if the appropriate button was pressed on the basis of the child's initial pointing gesture and if it occurred within 20 repetitions of the stimulus $(16 \mathrm{sec})$. A correct response resulted in the presentation of an 8-sec segment of the movie on the TV to which the child pointed. The child remained in the shaping phase until Experimenter 2 (inside the booth) determined that the child understood the task. At this point, Experimenter 2 gave the parent headphones that presented masking music, reaffirmed that the child understood the task, and left the child and parent alone in the booth.

During the testing phase, the same two full-vowel syllables used in the shaping phase were presented, but Experimenter 1 and the parent were blind to the stimulus presented on each trial. The subjects were required to reach a $90 \%$ correct criterion on at least 10 successive trials in the testing phase with the two full-vowel syllables before proceeding to the identification phase.

During the identification phase, the two full-vowel and the eight silent-center syllables were presented on separate trials to form a block of $\mathbf{1 0}$ trials (order of syllables was randomized across trials within each block). Only the two full-vowel syllables were differentially reinforced, whereas either a rightward or a leftward pointing response to the silent-center syllables was reinforced. Incorrect pointing responses to the full-vowel syllables resulted in immediate termination of the trial and no visual reward. The child was told prior to the identification phase that some of the sounds would be unusual, but to point to the TV with which the sound was associated during the shaping and testing phases.

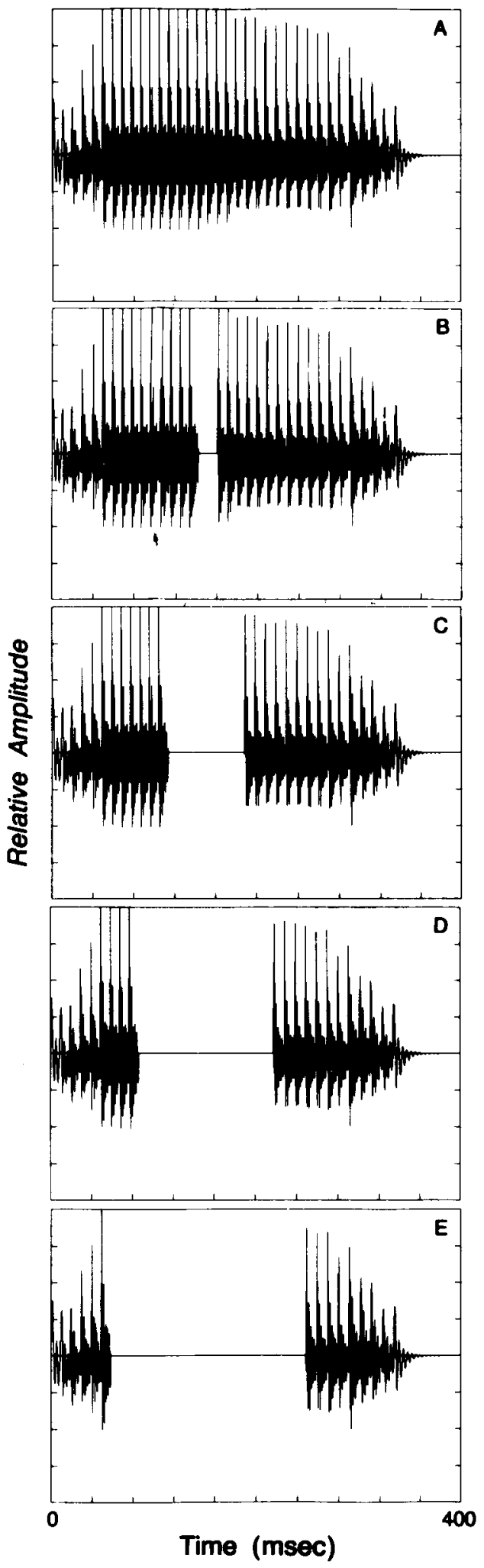

Figure 1. Waveforms of the five /bad/ stimuli used in Experiment 1: (A) full-vowel syllable, (B) 10\% silent-center syllable, (C) $35 \%$ silent-center syllable, (D) $65 \%$ silent-center syllable, and (E) $90 \%$ silent-center syllable. 
If a child's pointing responses fell below $75 \%$ correct for the two full-vowel syllables (e.g., by pointing incorrectly to two of the four full-vowel trials within two trial blocks), the computer automatically reverted from the identification phase to the testing phase and the child was required to reach the $90 \%$ correct criterion again before returning to the identification phase. These alternations between the identification and testing phases occurred in less than $15 \%$ of the sessions that provided usable data. When eight identification trial blocks (a total of 80 trials) had been completed, or the child showed signs of restlessness, the session was ended. The number of sessions required to complete 80 trials ranged from 3 to 5 , with a mean of 3.5 sessions per child.

\section{Results and Discussion}

The ability of the children and adults to identify the fullvowel and silent-center syllables correctly is illustrated in Figure 2. Although the adults performed nearly perfectly, the children showed considerably more variability. Nevertheless, the children's performance was significantly above chance for all five syllable pairs $(p<.01$ for all $t$ tests). Thus, although greater amounts of vowel deletion led to poorer identification performance $[F(4,40)$ $=3.12, p<.025$ ], 3-year-olds identified the vowels reliably even when $90 \%$ of the steady-state formant information was replaced with silence.

The ability of the children to identify the vowels correctly when $10 \%, 35 \%$, or $65 \%$ of the steady-state formant information was replaced with silence does not necessarily imply the use of dynamic formant information for vowel perception. In natural-speech CVC syllables, the offset frequencies of the initial formant transitions do not terminate abruptly into steady-state formant frequencies. Rather, there is a "blending" of formant frequencies into a quasi-steady-state formant structure. Not only did the synthetic stimuli used in the present experiment have more abrupt changes from the formant transitions to the steady-state formants, but they also contained truly steady-state formants. Thus, the children could have maintained correct responding to the $10 \%, 35 \%$, and $65 \%$ deletion syllables by interpolating across the gap in this steady-state formant structure. In addition, it is unclear

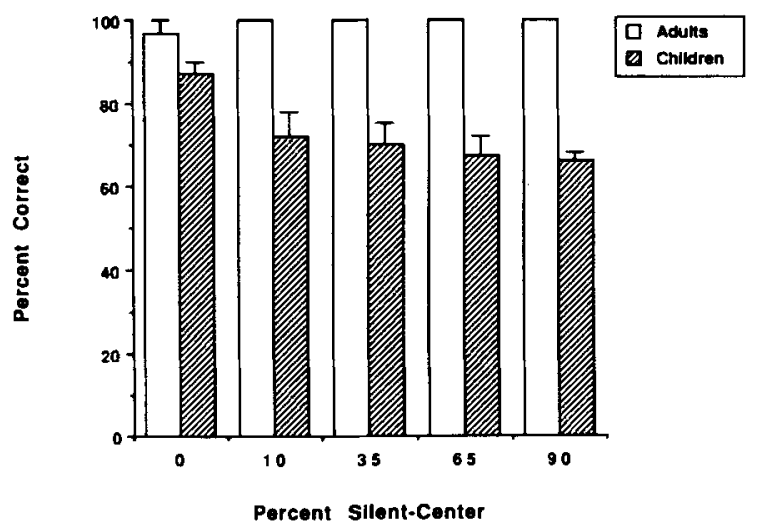

Figure 2. Mean percentage of correct identifications of the five stimuli for adults and children in Experiment 1. Error bars indicate 1 SEM.

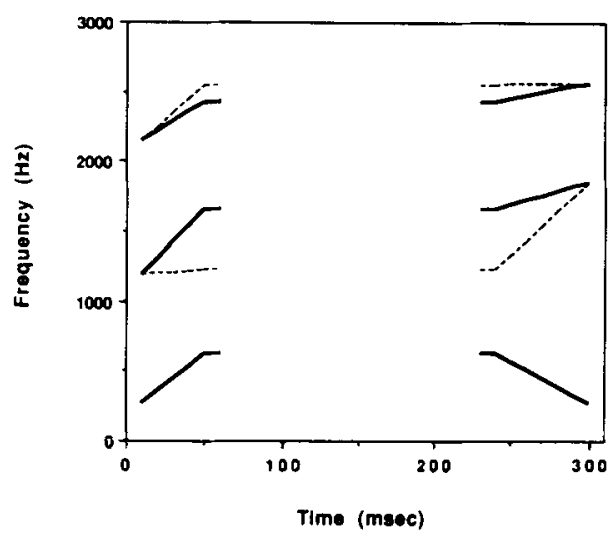

Figure 3. Schematic spectrograms of the $90 \%$ silent-center syllables. Solid lines indicate the edited / bad/ syllable and dashed lines indicate the edited / bad/syllable. The first formants were identical for the two syllables.

whether the children's vowel-identification performance would have been better or worse if the quasi-steady-state formant information present in natural CVC syllables had not been eliminated.

However, the children's ability to identify the vowels correctly when $90 \%$ of the steady-state formant information was eliminated suggests a more sophisticated form of formant-frequency interpolation. The steady-state formant information in these stimuli was carried by a single pitch period $(8.3 \mathrm{msec})$ at the offset of the syllable/initialformant transitions and at the onset of the syllable finalformant transitions (see Figure 3). Although one could argue that the children interpolated the formant structure of the "missing" vowel on the basis of these two pitch periods, it seems unlikely that the auditory system can extract steady-state formant information from a single pitch period.

A more plausible account for the interpolation process centers on the fact that the offsets of the syllable/initialformant transitions had the same frequencies as the onsets of the syllable/final-formant transitions (see Figure 3). Of course, there are a large number of possible quasisteady-state formant structures that could have occurred between the syllable/initial- and syllable/final-formant transitions (e.g., all three formants could have risen in frequency to values higher than the steady-state values of the full-vowel syllables). However, a "minimal distance" rule would lead to an interpolation that correctly matched the full-vowel syllables, particularly since the children had only two response categories (i.e., they could not report that the vowel was best described by /e/ or $/ \mathrm{l} /$ ) and there were no variations across tokens in voice quality (pitch or duration). Thus, the most parsimonious account of the children's above-chance performance for the $90 \%$ deletion syllables is that they extracted the differences in the second and third formant transitions from the full-vowel syllables and recognized the corresponding pattern of formant transitions when the steady-state formant information was eliminated from the silent-center syllables. 
Although this process of pattern-matching may not be identical to the mechanism used by adults (i.e., extraction of dynamic formant information), these results imply that children can make judgments of vowel category on the basis of very brief $(40-60$-msec) formant-transition differences.

Although as a group the children showed above-chance vowel-identification performance for all of the silentcenter syllables, an examination of the performance of individual children revealed that their results fell naturally into two subgroups (see Figure 4). The performance of one subgroup $(n=6)$ was above chance for all of the syllables, whereas the performance of the other subgroup $(n=5)$ was above chance for only the full-vowel syllables.

This poor performance by one subgroup of children when only $10 \%$ of the vowel (two pitch periods) was replaced with silence is surprising given the redundancy in the formant structure of the silent-center syllables and the fact that adults judged the $16.7-\mathrm{msec}$ gap to be only marginally noticeable. An ANOVA showed significant differences by both subgroup $[F(2,55)=97.0, p<.01]$ and stimulus condition $[F(4,55)=7.4, p<.01]$, as well as a significant subgroup $\times$ stimulus condition interaction $[F(8,55)=5.5, p<.01]$. The two subgroups showed no obvious differences on such factors as age, trials to reach testing criterion, or productive language skill (based on observation of the children's productions and parental descriptions of the children's language). Because nearly half of the children had great difficulty identifying some of the silent-center syllables correctly, it is possible that young children's use of dynamic formant information for vowel perception is just emerging at 3 years of age. The second experiment was an attempt to explore the finding that some children's performance fell to chance when as little as $10 \%$ of the steady-state formant information was deleted.

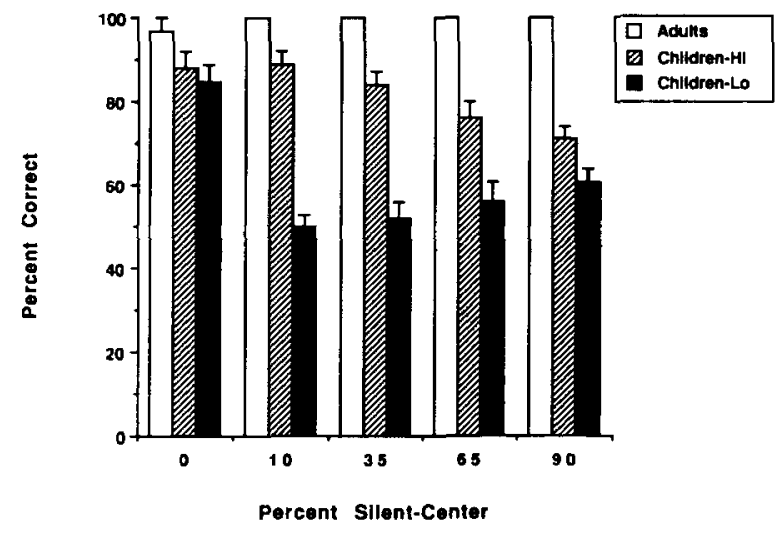

Figure 4. Mean percentage of correct identifications of the five stimuli for adults and the two subgroups of children in Experiment 1. Error bars = 1 SEM.

\section{EXPERIMENT 2}

In Experiment 1, each block of 10 trials during the identification phase consisted of the two full-vowel syllables and the eight silent-center syllables. Some children may have maintained correct responding by memorizing the full-vowel syllables during the shaping and testing phases and by pointing randomly to the silent-center syllables because reinforcement for these "unusual" stimuli was not contingent on a particular response. For example, if several of the silent-center syllables were presented on successive trials, some children may have become confused when an impulsive but incorrect point was reinforced. To reduce the possibility that high rates of noncontingent reinforcement biased some children to respond randomly to the silent-center syllables, the children in Experiment 2 received only two silent-center syllables ( $10 \%$ or $90 \%$ deletion) in each block of four trials during the identification phase. If the children were confused by the presence of the gap in the silent-center syllables and responded randomly to these stimuli, they would at most receive reinforcement on $75 \%$ of the trials, compared to the $90 \%$ possible in Experiment 1 . In addition, if a child forgot which TV was associated with the full-vowel syllables, no more than four successive trials without differential reinforcement (i.e., silent-center syllables) could occur before a full-vowel syllable with differential reinforcement was presented.

\section{Method}

Subjects. Three adults and 24 children, all recruited and tested as in Experiment 1, participated as subjects in the second experiment. All adults and 14 of the 24 children ( 7 in the $10 \%$ deletion condition and 7 in the $90 \%$ deletion condition) provided usable data. The successful subjects ranged in age from 36.0 to 45.0 months, with a mean age of 39.5 months. The other subjects were either unable to learn the task $(n=7)$ or dropped out because of illness or scheduling conflicts $(n=3)$.

Stimuli. All children were trained on the two full-vowel syllables used in Experiment 1. During the identification phase, children in the $10 \%$ deletion condition received 10 blocks of four stimuli consisting of the two full-vowel syllables and the two $10 \%$ silentcenter syllables. The children in the $90 \%$ deletion condition received 10 blocks of four stimuli consisting of the two full-vowel syllables and the two $90 \%$ silent-center syllables.

Apparatus and Procedure. The apparatus and procedure were the same as in Experiment 1. The number of sessions required to complete the full 40 trials ranged from 2 to 3 , with a mean of 2.1 sessions per child.

\section{Results and Discussion}

As shown in Figure 5, the pattern of results from Experiment 1 was replicated in that the performance of children in both the $10 \%$ and the $90 \%$ deletion conditions was either adult-like or at chance. On the group means, $t$ tests revealed no significant difference between the adults and the higher scoring children [for $10 \%$ silent-center sylkables, $t(5)=2.34$, n.s.; for $90 \%$ silent-center syllables, 


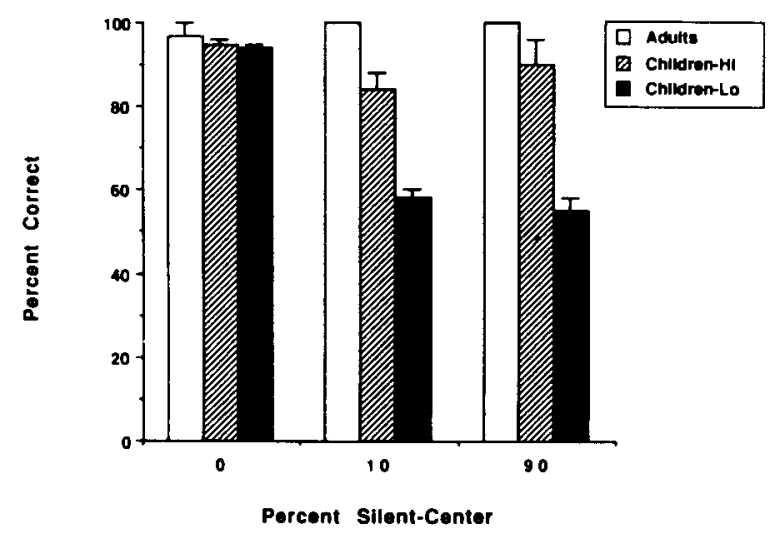

Figure 5. Mean percentage of correct identifications of the three stimuli for adults and the two subgroups of chlldren in Experiment 2. Error bars = 1 SEM.

$t(4)=1.31$, n.s.]. However, there was a significant difference between the two subgroups of children on the $10 \%$ silent-center syllables $[t(5)=3.43, p<.05]$ and also on the $90 \%$ silent-center syllables $[t(5)=5.00$, $\mathrm{p}<01$ ].

An important aspect of these results is the fact that 3 of the 7 children in the $90 \%$ deletion condition performed near adult levels, replicating the finding from Experiment 1 that vowel identification can be maintained despite the absence of nearly all steady-state formant information. The fact that 4 of the 7 children in the $90 \%$ deletion condition performed at chance is not surprising, as vowel identification even for adults in this condition was quite difficult. Moreover, the performance difference between the subgroups in both the $10 \%$ and the $90 \%$ deletion conditions cannot be attributed to a general task variable since no significant between-group differences in performance on the full-vowel syllables were present $[t(16)=.77$, n.s.]. The surprising aspect of the data shown in Figure 5 was the chance performance of 2 of the 7 children in the $10 \%$ deletion condition. These children also performed very well on the full-vowel syllables but very poorly when only two pitch periods $(16.7 \mathrm{msec})$ of the steady-state vowel were replaced with silence.

\section{EXPERIMENT 3}

One possible source of confusion that might have led to the random performance by 2 of the 7 children in the $10 \%$ silent-center condition of Experiment 2 was the presence of a 16.7-msec gap in the middle of the syllable. To reduce this gross spectral cue, syllables were created in which the gap was filled with noise. ${ }^{1}$ Although Jenkins et al. (1983) reported that vowel identification in adults was poorer for noise-center syllables than for silentcenter syllables, the magnitude of this effect was relatively small (18.7\% vs. $7.6 \%$ error rate) and the noise accounted for $50 \%-65 \%$ of the total syllable duration. Parker and Diehl (1984) reported that the error rate for adults' vowel identification of noise-center syllables was greater than for silent-center syllables only when more than $80 \%$ of the syllable was modified. Thus, any masking of dynamic formant information by the noise should be minimal when only $10 \%$ of the middle of the syllable is modified. The substitution of noise for the gap in the middle of the $10 \%$ silent-center syllables was expected to provide children with a less spectrally discrepant stimulus, thereby facilitating identification of the vowels compared to the silentcenter syllables used in Experiments 1 and 2.

\section{Method}

Subjects. Subjects were recruited as in the previous two experiments. Three adults and 17 children participated, but only 7 children successfully completed the task. Seven children were unable to learn the task; 3 others left the study because of illness. The age range of successful subjects was 37.5 to $\mathbf{4 4 . 0}$ months, with a mean age of $\mathbf{4 0 . 4}$ months.

Stimuli. The full-vowel syllables from Experiment 1 were used as the training stimuli. To create the modified tokens, noise was inserted into the full-vowel syllables using two waveform-editing systems (Bernacki, 1981; Luce \& Carrell, 1981), thereby replacing the central $10 \%$ of the steady-state formant information. Sample waveforms are illustrated in Figure 6.

Apparatus and Procedure. The apparatus and procedure were the same as in Experiments 1 and 2. The number of sessions required to complete 40 trials ranged from 1 to 4 , with a mean of 2.0 sessions per child.

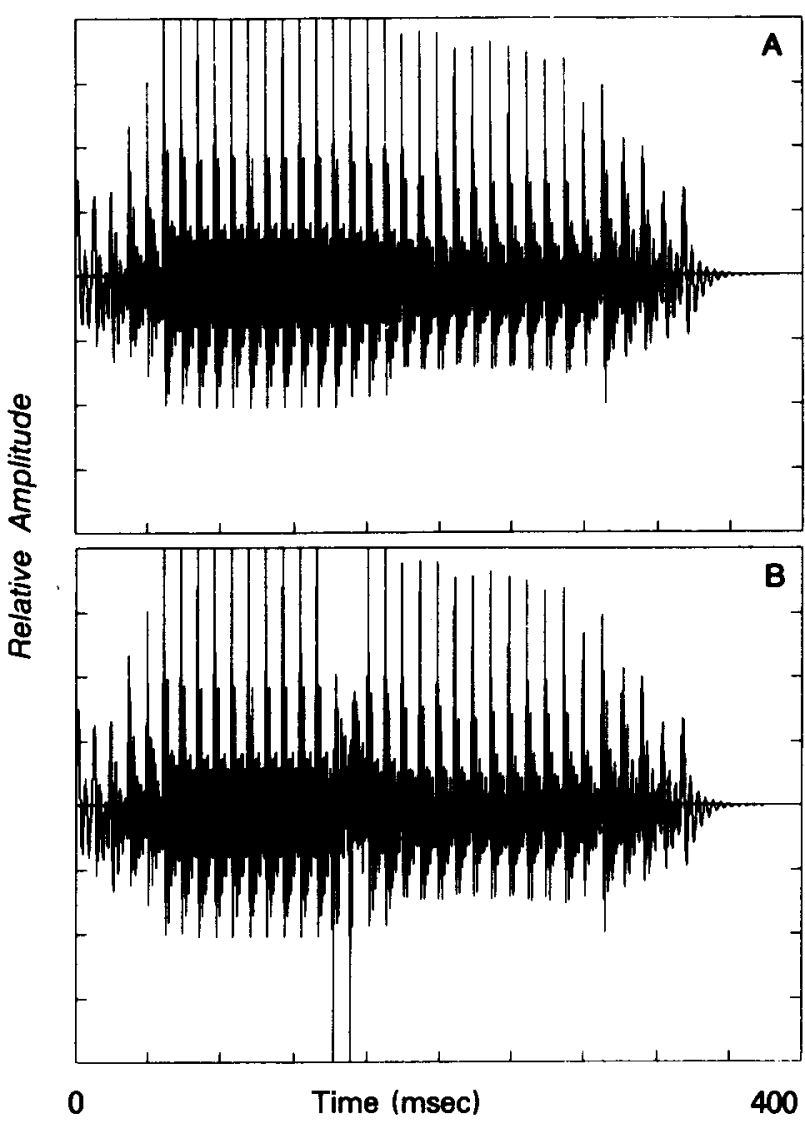

Figure 6. Waveforms of the two /bæd/ stimuli used in Experiment 3: (A) full-vowel syllable, and (B) $10 \%$ noise-center syllable in which the central two pitch periods are replaced with noise. 


\section{Results and Discussion}

As shown in Figure 7, the performance of the children did not differ significantly from the performance of the adults, either for the full-vowel syllables $[t(7)=.44$, n.s. $]$ or for the $10 \%$ noise-center syllables $[t(7)=1.76$, n.s. $]$. Moreover, all 7 of the children performed well on the $10 \%$ noise-center syllables (identification performance ranged from $85 \%-100 \%$ correct). These results suggest that the less discrepant spectral change associated with the $10 \%$ noise-center syllables, relative to the $10 \%$ silent-center syllables, prevented some children from responding randomly to the noise-center stimuli. However, one might argue that the small sample size of children tested in Experiment 3 decreased the likelihood of uncovering a subgroup of children whose performance fell to chance in the $10 \%$ noise-center condition (although a similar sample size in Experiment 2 revealed 2 children whose performance fell to chance in the $10 \%$ silent-center condition)

\section{EXPERIMENT 4}

To provide a stronger test of the hypothesis that noisecenter syllables are less likely to disrupt children's perception of vowels than are silent-center syllables, both silent-center and noise-center syllables were presented to the same children. If some children are more distracted by silent-center than by noise-center syllables, then those children should perform poorly on silent-center syllables, but no children should perform poorly on noise-center syllables.

\section{Method}

Subjects. The subjects were 3 adults and 32 children recruited as in the previous studies but tested on a maximum of two sessions. ${ }^{2}$ Due to a number of factors that influenced subject availability, only 15 children completed the task successfully. Three subjects left the study because of illness and 14 others were unable to complete the task within the time allotted. The successful subjects ranged in age from 38.0 to 42.0 months, with a mean age of 40.4 months.

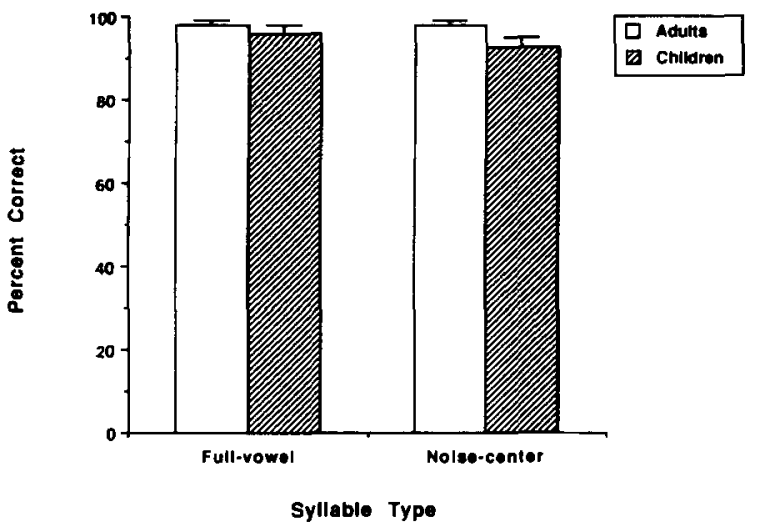

Figure 7. Mean percentage of correct identifications of the two stimuli for adults and children in Experiment 3. Error bars $=1$ SEM.

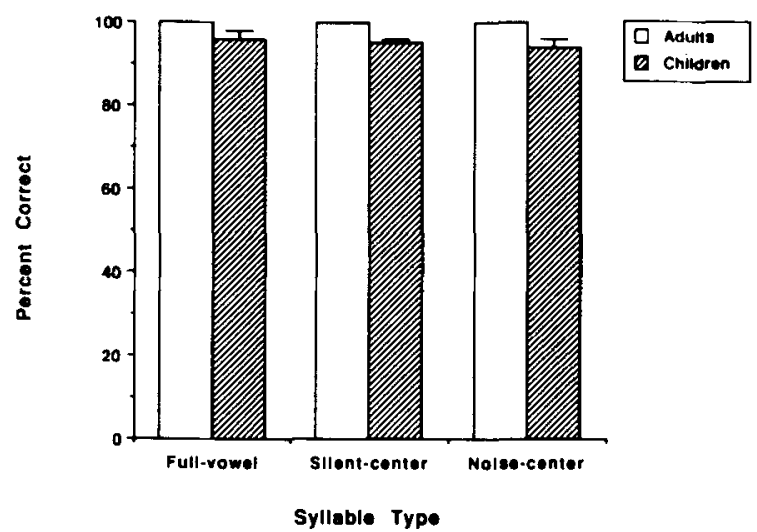

Figure 8. Mean percentage of correct identifications of the three stimulf for adults and children in Experiment 4. Error bars = 1 SEM.

Stimuli. The stimuli were the full-vowel and $10 \%$ silent-center syllables from Experiments 1 and 2 and the 10\% noise-center syllables from Experiment 3.

Apparatus and Procedure. The apparatus and procedure were the same as in the three previous experiments, except that the subjects were restricted to two visits to the lab, which prevented 14 subjects from completing the entire task. Each block of six trials consisted of the two full-vowel, the two $10 \%$ silent-center, and the two $10 \%$ noise-center syllables. Each child who completed the task received a total of 10 blocks (60 trials).

\section{Results and Discussion}

In contrast to Experiments 1 and 2, none of the children performed poorly on the $10 \%$ silent-center stimuli and well on the $10 \%$ noise-center stimuli. As shown in Figure 8, adults and children performed equally well on the full-vowel syllables and on the noise- and silent-center syllables $[F(2,17)=3.02$, n.s. $]$.

These results are somewhat puzzling, in that several children performed poorly in Experiments 1 and 2 in the $10 \%$ silent-center condition, whereas none of the children performed poorly in Experiment 3 in the $10 \%$ noise-center condition. One possible explanation for this apparent discrepancy is that the children in Experiment 4, who heard both the noise-center and the silent-center syllables in the identification phase, were able to infer more easily that the task required a judgment of vowel category rather than a decision about the absolute identity of the modifiedvowel syllables compared to the full-vowel syllables. That is, some of the children in Experiments 1 and 2 may have become confused by the fact that the silent-center syllables did not match the full-vowel training stimuli exactly. Because their initial "guess" at which pointing response was appropriate for the silent-center syllables resulted in reinforcement, they may have inferred that it was not necessary to make a similarity judgment between the silentcenter and the full-vowel syllables during the identification phase. Apparently, the reduced spectral discrepancy of the noise-center syllables lowered the probability that the children would become confused by the absence of an exact match between the noise-center and the full-vowel 
syllables, thereby enabling all children in Experiment 3 to make appropriate similarity judgments. Moreover, the intermixing of noise-center with silent-center syllables in Experiment 4 apparently increased the probability that the children would recognize that the task did not require exact matches between the silent- and noise-center syllables and the full-vowel syllables in the identification phase.

\section{GENERAL DISCUSSION}

The present series of experiments provided three new contributions to the area of speech perception in young children. First, the modified two-alternative pointing technique was shown to be suitable for gathering identification data from children as young as 3 years of age. Although presentation of the generalization stimuli under conditions of nondifferential reinforcement rather than during extinction of reinforcement appeared to confuse some of the children, all children were able to make similarity judgments of the generalization stimuli unless these stimuli were grossly discrepant from the training stimuli. Thus, the pointing technique may be particularly appropriate for gathering identification data from children who are presented with tokens from a synthetic speech continuum (e.g., variations in voice onset time). Moreover, by training children with more than a single exemplar of each stimulus, it may be possible to reduce the probability that some children will interpret the task as one that requires an exact match with one of the training stimuli during the identification phase.

Second, the results of the first two experiments demonstrated that many 3-year-olds can identify vowels correctly in the context of synthetic CVC syllables, even when as much as $90 \%$ of the steady-state formant information has been replaced with silence. Although these results are not a definitive demonstration that children use dynamic formant information for vowel identification, the results from the $90 \%$ deletion condition suggest that children are capable of interpolating vowel targets from a pair of 40-60msec formant transitions. This ability to interpolate the identity of vowels from limited spectral information may be of considerable value when listening to fluent speech in which quasi-steady-state formant frequencies are reduced or are absent.

Third, the pattern of results from all four experiments suggests that children are remarkably sensitive to brief spectral changes embedded within CVC syllables. Several children in Experiments 1 and 2 failed to generalize their responding from the full-vowel training syllables to the $10 \%$ silent-center syllables. Thus, although these children did not perform like adults, their failure to generalize indicated that it would be possible to assess the excellent discriminative abilities of 3-year-olds using a modified version of the pointing procedure (e.g., by training the children to respond to a single target stimulus vs. all possible nontarget stimuli). In this way, one could determine whether children who generalize their responding to spectrally similar stimuli (e.g., the $10 \%$ noise-center syllables) are in fact generalizing on the basis of a similarity judgment or are simply failing to discriminate the generalization stimuli from the training stimuli.

Finally, it remains unclear on what basis adults identify vowels in vowelless CVC syllables. Although it appears that adults can base their identification of vowels in silent-center and noise-center syllables on dynamic formant information, it is unclear whether the critical information is contained in the formant transitions themselves or in the formant-frequency targets interpolated from the trajectories of the formant transitions. Although there are limitations to the use of synthetic speech in studying vowel perception in "natural" contexts, an understanding of the minimal information required for vowel perception may be revealed only by careful manipulations of synthetic speech.

\section{REFERENCES}

BERNACKI, B. (1981). WAVMOD: A program to modify digital waveforms (Progress Rep. No. 7). Bloomington: Indiana University Speech Research Laboratory.

Delattre, P., Liberman, A. M., Cooper, F. S., a Gerstman, L. J. (1952). An experimental study of the acoustic determinants of vowel color: Observations on one- and two-formant vowels synthesized from spectrographic patterns. Word, 8, 195-210.

Diehl, R. L., McCusker, S. B., \& Chapman, L. S. (1981). Perceiving vowels in isolation and in consonantal context. Journal of the Acoustical Society of America, 69, 239-248.

Jenkins, J. J., Strange, W., \& Edman, T. R. (1983). Identification of vowels in "vowelless" syllables. Perception \& Psychophysics, 34, 441-450.

Joos, M. A. (1948). Acoustic phonetics. Language Supplement, 24, 1-136.

KLATT, D. H. (1980). Software for a cascade/parallel formant synthesizer. Joumal of the Acoustical Society of America, 67, 971-995.

Kubaska, C. A., AsLIN, R. N. (1985). Categorization and normalization of vowels by 3 -year-old children. Perception \& Psychophysics, 37, 355-362.

KuHL, P. K. (1979). Speech perception in early infancy: Perceptual constancy for spectrally dissimilar vowel categories. Journal of the Acoustical Society of America, 66, 1668-1679.

KuHL, P. K. (1983). Perception of auditory equivalence classes for speech in early infancy. Infant Behavior \& Development, 6, 263-285.

KUWABARA, H. (1985). An approach to normalization of caarticulation effects for vowels in connected speech. Journal of the Acoustical Society of America, 77, 686-694.

Luce, P. A., \& CARrell, T. D. (1981). Creating and editing waveforms using WAVES (Progress Rep. No. 7). Bloomington: Indiana University Speech Research Laboratory.

O’Malley, M. H., Peterson, G. E. (1966). An experimental method for prosodic analysis. Phonetica, 15, 1-13.

PARKer, E. M., \& Diehl, R. L. (1984). Identifying vowels in CVC syllables: Effects of inserting silence and noise. Perception \& Psychophysics, 36, 369-380.

Peterson, G. E., Barney, H. L. (1952). Control methods used in a study of the vowels. Joumal of the Acoustical Society of America, 24, 175-184.

Strange, W., Jenkins, J. J., Johnson, T. L. (1983). Dynamic specification of coarticulated vowels. Journal of the Acoustical Society of America, 74, 695-705. 
Strange, W., Verbrugge, R. R., Shankweiler, D. P., \& Edman, T. R. (1976). Consonant environment specifies vowel identity. Journal of the Acoustical Society of America, 60, 213-224.

Verbrugge, R. R., Strange, W., Shankweiler, D. P., \& Edman, T. R. (1976). What information enables a listener to map a talker's vowel space? Journal of the Acoustical Society of America, 60, 198-212.

\section{NOTES}

1. The method developed by O'Malley and Peterson (1966) was used to generate the noise. Their algorithm preserves the average frequency and duration of the deleted vowel segment while randomizing the spectral information.

2. Because several children in the first three experiments who were tested up to five times provided no usable data, and most of the successful subjects provided data in three or fewer sessions, the number of times each child visited the lab was limited to two if the child had not yet succeeded in meeting the testing-phase criterion. This restriction may have prevented some children who would otherwise have provided usable data from reaching the identification phase.

(Manuscript received August 12, 1988; revision accepted for publication March 19, 1989.) 